Brit. Heart F., 1966, 28, 62.

\title{
Corrected (Frank), Uncorrected (Cube), and Standard Electrocardiographic Lead Systems in Recording Augmented Right Ventricular Forces in Right Ventricular Hypertension ${ }^{\star}$
}

\author{
R. GAMBOA $†$, P. G. HUGENHOLTZ, AND A. S. NADAS \\ From the Sharon Cardiovascular Unit, Children's Hospital Medical Center, and the Department of Pediatrics of \\ Harvard Medical School, Boston, Massachusetts, U.S.A.
}

In the clinical detection of right ventricular hypertrophy, the electrocardiogram traditionally has been the most useful guide. Yet the fact that no unanimity exists in regard to the electrocardiographic criteria for this diagnosis suggests a lack of specificity of the method. In fact, in some series, with right ventricular hypertrophy proven at necropsy, the accuracy of the electrocardiographic diagnosis has been found to be wanting in many instances (Scott, 1960). The introduction of "uncorrected" vectorcardiographic lead systems (Wilson, Johnston, and Kossmann, 1947; Duchosal and Sulzer, 1949; Grishman and Scherlis, 1952), with a new set of criteria, has led, initially at least, to considerable enthusiasm about improved diagnostic accuracy (Donoso et al., 1955). The subsequent development of "corrected" vectorcardiographic lead systems (Schmitt and Simonson, 1955; Frank, 1956; McFee and Parungao, 1961) promised a further improvement in the assessment of right ventricular hypertrophy (Hugenholtz and Gamboa, 1964), but detailed studies of this problem by these new techniques have thus far been lacking.

In order to evaluate the relative merits of the most frequently used lead systems (standard electrocardiogram, cube vectorcardiogram, Frank vectorcardiogram), each was applied in quick succession to patients with proven right ventricular hyperten-

Received April 12, 1965.

* Supported in part by grants HE-52515-06, HTS5310, and FF-308, National Institutes of Health, Bethesda, Maryland, U.S.A.

† Present address: Department of Pediatrics, Southwestern Medical School, University of Texas, Dallas, Texas-75235, U.S.A. sion. Only patients with congenital valvar pulmonary stenosis were subjected to this analysis in an effort to study only the effects of concentric hypertrophy of the right ventricle.

\section{SuBJECT AND METHOdS}

The group consisted of 50 patients with congenital valvar pulmonary stenosis, aged from $2 \frac{1}{2}$ to 23 years. There were 25 female and 25 male subjects. All were studied by cardiac catheterization and the electrocardiographic and vectorcardiographic records were obtained shortly before or after the catheterization procedure. Details of the latter have been given before (Hugenholtz and Gamboa, 1964). All studies were done under resting circumstances after premedication with a meperidine-phenergan-chlorpromazine compound. Cine-angiograms or biplane angiograms were obtained in all patients to confirm the diagnosis. From cardiac output determinations, immediately preceding or following the recording of right ventricular and pulmonary artery pressures, valve area and stroke work were calculated.

In 30 of these patients both cube and Frank vectorcardiograms were recorded in quick succession as previously described (Hugenholtz and Gamboa, 1964). In the 20 remaining patients either the cube or the Frank systems were used. The maximum rightward spatial vector (MRSV) was derived from each by Pythagoras' theorem, MRSV $=\sqrt{X^{2}+Y^{2}+Z^{2}}$, where $X, Y, Z$ represent the transverse, vertical, and sagittal components of that vector. Similarly a sum of spatial vectors (SMRSV) was obtained by adding the magnitude of the MRSV to that of the vector occurring $0.005 \mathrm{sec}$. before and to those occurring 0.005 and $0.010 \mathrm{sec}$. after the maximum vector. In effect, this measurement is representative of a cone removed from the spatial 62 
ventricle may be considered to exert a dominant influence. This measurement was made irrespective of the moment of the QRS time it occupied. The ratio of the magnitude of the maximum leftward to the maximum rightward vector, as projected on the transverse axis of the frontal plane, was also determined ( $L / R$ ratio).

In addition, the time when the MRSV occurred as well as when the maximum spatial vector was inscribed was determined. Analysis of direction of rotation and of differences in morphology between Frank and cube loops were also studied.

The standard 12 lead electrocardiogram was obtained in the usual fashion in all 50 patients. The amplitude of the $R$ in $V 1$ and the $R / S$ ratio in V1 were selected as generally accepted criteria for right ventricular hypertrophy. In addition, a sum of amplitudes consisting of $R$ in $\mathrm{V} 1+\mathrm{S}$ in $\mathrm{V} 6+\mathrm{S}$ in standard lead I was calculated in an effort to add to the standard electrocardiographic criteria one that would be more representative of spatial forces.

Correlations were then carried out between the hæmodynamic data [right ventricular peak pressure (RVPP), pulmonary valve area, stroke work, and systemic pressure/RVPP ratio], the vectorcardiographic data [MRSV, SMRSV and $L / R$ ratio (derived by both vectorcardiographic systems)], and the three elctrocardiographic criteria outlined above.

In order to assess the statistical significance of the results in the 30 patients studied with both vectorcardiographic systems, the same analyses were also carried out for the entire group of 50 patients. Details of the vectorcardiographic or hæmodynamic findings in the 20 patients with only one vectorcardiographic system are not given in this report but are available upon request to the authors.

\section{RESULTS}

All pertinent data in the 30 patients are given in Table I, arranged in order of increasing ventricular pressures. Right ventricular hypertension was present in all patients and ranged from 38 to 196 $\mathrm{mm}$. $\mathrm{Hg}$ peak systolic pressure at rest. Cardiac index was within normal limits in 20 (2.5 to 4.5 $1 . / \mathrm{min} . \mathrm{m}^{2}{ }^{2}$ ) but was slightly raised in 10 patients, 9 of whom showed mild increases in heart rate at the time of study. A small atrial right-to-left shunt was present in 3 patients. In these, the cardiac index given represents pulmonary flow.

Pulmonary valve areas ranged from $2.00 \mathrm{~cm} .{ }^{2}$ to $0.05 \mathrm{~cm}^{2}$ per patient. Right ventricular stroke work progressively increased as the severity of the stenosis increased. Cine-angiocardiography confirmed the valvar nature of the obstruction in all and showed additional infundibular stenosis in 8 patients.

The vectorcardiographic measurements showed a gradual increase in magnitude of the MRSV and SMRSV parallel to the increase in right ventricular pressure. The MRSV as determined from the Frank system ranged from 0.77 to $3.31 \mathrm{mV}$. The SMRSV ranged from $3.16 \mathrm{mV}$ to $12.52 \mathrm{mV}$. The magnitude of these measurements was much smaller in the data recorded by the cube system, 0.34 to $1.87 \mathrm{mV}$ for MRSV and 1.58 to $5.76 \mathrm{mV}$ for SMRSV. In contrast the $L / R$ ratios were quite similar in both systems. The electrocardiographic data were variable and did not show the gradual increase in magnitude paralleling the increase in right ventricular hypertension.

A linear relation was found between MRSV (Frank) and right ventricular peak pressure (RVPP), $r=0.85, p<0.001$, standard error of the estimate (S.E.E.) $30 \mathrm{~mm}$. $\mathrm{Hg}$. The regression equation applicable to the data derived by the Frank system is $R V P P=60.48 \mathrm{mV}+0.22$. For the cube data $\mathrm{r}=0.80, \mathrm{p}<0.001$, and S.E.E. was $37 \mathrm{~mm}$. $\mathrm{Hg}$ (Fig. 1). Since in this and all other comparisons the correlation coefficient derived from the Frank system data exceeded those from the cube system data, regression equations for the latter were not calculated. Among the electrocardiographic data, the measurement of the $S$ in lead $I+R$ in $V 1+S$ in V6 gave the best correlation from any of the electrocardiographic measurements, but was still below the VCG correlations, $r=0.60, p<0.001$, S.E.E. $45 \mathrm{~mm}$. $\mathrm{Hg}$ (Fig. 2). When the number of observations was increased to 50 , the correlation coefficients were 0.87 (Frank), 0.82 (cube), and 0.64 (ECG), while S.E.E. remained virtually unchanged at 28,32 , and $50 \mathrm{~mm}$. $\mathrm{Hg}$, respectively.

Each of the conventional ECG criteria gave a much lower correlation. For the relation between the amplitude of $R$ in V1 and RVPP, $r$ was 0.57 , S.E.E. $50 \mathrm{~mm}$. $\mathrm{Hg}(\mathrm{n}=30$, Fig. 2), while adding $R$ in V1 to $S$ in V6 decreased the calculated coefficient to $0.30(n=50)$. The $R / S$ ratio in $V 1$ also gave an insignificant correlation with RVPP, $r=0 \cdot 12$.

A more ideal correlation, again showing a linear relation, was found between SMRSV and RVPP (Fig. 3). For the Frank data, $r=0.90, p<0.001$, S.E.E. $23 \mathrm{~mm}$. $\mathrm{Hg}$, and for the cube data, the correlation coefficient was $0.83, \mathrm{p}<0.001$, S.E.E. 32 $\mathrm{mm}$. Hg. The regression equation for the Frank findings is $R V P P=18.0 \mathrm{mV}-12$. When the larger group $(n=50)$ was used, again the $r$ value changed little, 0.90 and 0.82 respectively, but the S.E.E. decreased further to 20 and $30 \mathrm{~mm}$. $\mathrm{Hg}$, respectively. Thus the chief effect of the increase in the number of observations was a decrease in S.E.E., while the correlation coefficient remained essentially unchanged from that found in the smaller, truly comparable, group.

There was a satisfactory relation between the $L / R$ ratio and the RVPP (Fig. 4). In contrast to the 
TABLE

HÆMODYNAMIC, VECTORCARDIOGRAPHIC,

\begin{tabular}{|c|c|c|c|c|c|c|c|c|c|c|c|}
\hline \multirow{4}{*}{$\begin{array}{l}\text { Case No., } \\
\text { sex, and age } \\
\text { (yr.) }\end{array}$} & \multicolumn{6}{|c|}{ Hæmodynamic data } & \multirow{2}{*}{\multicolumn{5}{|c|}{$\frac{\text { Vectorcardiographic and }}{\text { Frank }}$}} \\
\hline & \multirow{3}{*}{ BSA m.2 } & \multirow{3}{*}{$\underset{\left(1 . / \mathrm{min} . \mathrm{m} \cdot{ }^{2}\right)}{\mathrm{CI}}$} & \multirow[t]{3}{*}{ HR } & \multirow{3}{*}{$\underset{\text { beat m./2) }}{\mathrm{SI}}$} & \multirow{3}{*}{$\underset{(\mathrm{mm} . \mathbf{H g})}{\text { RVSP }}$} & \multirow{3}{*}{$\underset{(\mathrm{mm} . \mathrm{Hg})}{\mathrm{SAP}}$} & & & & & \\
\hline & & & & & & & \multicolumn{4}{|c|}{ Magnitude of spatial vectors } & \multirow{2}{*}{$\begin{array}{c}\text { Direction } \\
\text { of } \\
\text { rotation }\end{array}$} \\
\hline & & & & & & & MSV & MRSV & $\begin{array}{l}\mathrm{L} / \mathrm{R} \\
\mathrm{mV} \\
\text { ratio }\end{array}$ & $\begin{array}{l}\text { Sum } \\
\text { of } \\
\text { vectors }\end{array}$ & \\
\hline $\begin{array}{lll}1 & F & 16 \frac{1}{2} \\
2 & M & 16 \\
3 & M & 6 \\
4 & M & 11 \\
5 & F & 12 \\
6 & M & 15 \\
7 & F & 5 \\
8 & M & 10 \\
9 & F & 10 \\
10 & F & 11 \\
11 & M & 3 \\
12 & F & 5 \\
13 & F & 1 \frac{8}{18} \\
14 & F & 2 \frac{1}{2} \\
15 & M & 4 \\
16 & F & 6 \\
17 & M & 11 \\
18 & F & 26 \\
19 & F & 9 \\
20 & M & 14 \\
21 & F & 5 \frac{1}{2} \\
22 & F & 8 \\
23 & F & 5 \\
24 & F & 10 \\
25 & M & 14 \\
26 & F & 1 \frac{1}{2} \\
27 & M & 2 \\
28 & M & 13 \frac{1}{2} \\
29 & M & 20 \\
30 & F & 4 \frac{1}{2}\end{array}$ & $\begin{array}{l}1.53 \\
1.80 \\
0.92 \\
1.21 \\
1.12 \\
1.73 \\
0.36 \\
1.05 \\
1.13 \\
1.09 \\
0.60 \\
1.00 \\
0.41 \\
0.53 \\
0.55 \\
0.83 \\
1.04 \\
1.80 \\
1.16 \\
1.12 \\
0.75 \\
0.95 \\
0.76 \\
0.97 \\
1.00 \\
0.47 \\
0.44 \\
1.35 \\
1.80 \\
0.68\end{array}$ & $\begin{array}{l}5.9 \\
2.9 \\
5.0 \\
4 \cdot 2 \\
4 \cdot 2 \\
3.9 \\
8.1 \\
4.8 \\
5.2 \\
3.9 \\
5.0 \\
4.6 \\
4.0 \\
3.4 \\
3.1 \\
5.9 \\
2.7 \\
4 \cdot 0 \\
5.9 \\
4.3 \\
3.6 \\
3.5 \\
2.3 \\
2.4 \\
5.9 \\
1.6 \dagger \\
3.0 \dagger \\
2.8 \\
3.0 \\
3.3 \dagger\end{array}$ & $\begin{array}{r}96 \\
72 \\
72 \\
80 \\
80 \\
66 \\
100 \\
88 \\
81 \\
74 \\
82 \\
90 \\
100 \\
90 \\
88 \\
100 \\
77 \\
86 \\
88 \\
85 \\
100 \\
105 \\
74 \\
90 \\
88 \\
100 \\
100 \\
78 \\
60 \\
100\end{array}$ & $\begin{array}{l}61 \\
40 \\
69 \\
53 \\
53 \\
59 \\
81 \\
54 \\
64 \\
53 \\
47 \\
51 \\
79 \\
38 \\
35 \\
59 \\
33 \\
47 \\
67 \\
51 \\
36 \\
33 \\
31 \\
27 \\
68 \\
16 \\
35 \\
39 \\
60 \\
33\end{array}$ & $\begin{array}{l}38 \\
40 \\
46 \\
54 \\
54 \\
64 \\
75 \\
78 \\
79 \\
82 \\
85 \\
87 \\
87 \\
90 \\
90 \\
90 \\
100 \\
100 \\
100 \\
106 \\
125 \\
132 \\
160 \\
180 \\
180 \\
180 \\
180 \\
190 \\
190 \\
196\end{array}$ & $\begin{array}{l}130 / 70 \\
113 / 57 \\
105 / 70 \\
110 / 70 \\
110 / 8(\mathrm{LV}) \\
115 / 62 \\
125 / 65 \\
125 / 75 \\
140 / 75 \\
116 / 60 \\
100 / 40 \\
100 / 58 \\
92 / 40 \\
110 / 56 \\
100 / 45 \\
100 / 60 \\
140 / 60 \\
120 / 80 \\
140 / 75 \\
130 / 72 \\
115 / 90 \\
115 / 60 \\
100 / 50 \\
120 / 68 \\
112 / 68 \\
88 / 50 \\
100 / 42 \\
148 / 88 \\
104 / 68 \\
128 / 64\end{array}$ & $\begin{array}{l}2.01 \\
2 \cdot 16 \\
1.87 \\
1.94 \\
2.08 \\
1.87 \\
1.80 \\
2.08 \\
1.80 \\
2.11 \\
1.88 \\
2 \cdot 44 \\
1.61 \\
1.58 \\
2.13 \\
2.16 \\
1.80 \\
1.72 \\
1.77 \\
1.74 \\
1.49 \\
1.88 \\
2.44 \\
2.59 \\
2.95 \\
3.35 \\
2.59 \\
2.44 \\
3.00 \\
2.65\end{array}$ & $\begin{array}{l}0.89 \\
1.26 \\
1 \cdot 15 \\
1 \cdot 15 \\
0.77 \\
1.72 \\
1.22 \\
1.22 \\
1 \cdot 72 \\
2.04 \\
1.94 \\
1.58 \\
1.58 \\
1.44 \\
2 \cdot 10 \\
2.01 \\
1.80 \\
1.72 \\
1.77 \\
1.74 \\
1.48 \\
1.87 \\
2.44 \\
2.59 \\
2.95 \\
3.31 \\
2.59 \\
2.44 \\
3.02 \\
2.63\end{array}$ & 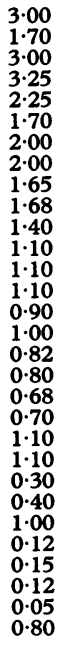 & 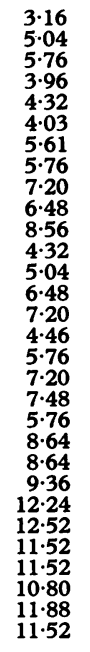 & $\begin{array}{l}\text { CCW } \\
C C W \\
C C W \\
C C W \\
C C W \\
C C W \\
C C W \\
C C W \\
C C W \\
C W \\
C W \\
C C W \\
C W \\
C W \\
C W \\
C W \\
C W \\
\text { Fig. } 8 \\
C W \\
C W \\
C W \\
C C W \\
C W \\
C W \\
\text { Fig. } 8 \\
C W \\
\text { CW } \\
\text { CW } \\
\text { Fig. } 8 \\
\text { CW }\end{array}$ \\
\hline
\end{tabular}

* CI, cardiac index; HR, heart rate; SI, stroke index; RVSP, right ventricular systolic pressure; SAP, systemic artery † Small right-to-left shunt at atrial level.

spatial measurements, there was little difference between the Frank and the cube data, $r=0.83$, $\mathrm{p}<0.001$, and $\mathrm{r}=0.82, \mathrm{p}<0.001$, respectively. The regression equation for the Frank data is $\mathrm{RVPP}=-95(\log \mathrm{L} / \mathrm{R}+1)+200$. There was also a direct linear relation between the $L / R$ ratio and the systemic pressure/RVPP ratio, $\mathrm{r}=0.82, \mathrm{p}<0.001$ for the Frank system (Fig. 5).

Correlation of SMRSV and MRSV with pulmonary valve area (PVA) was also carried out

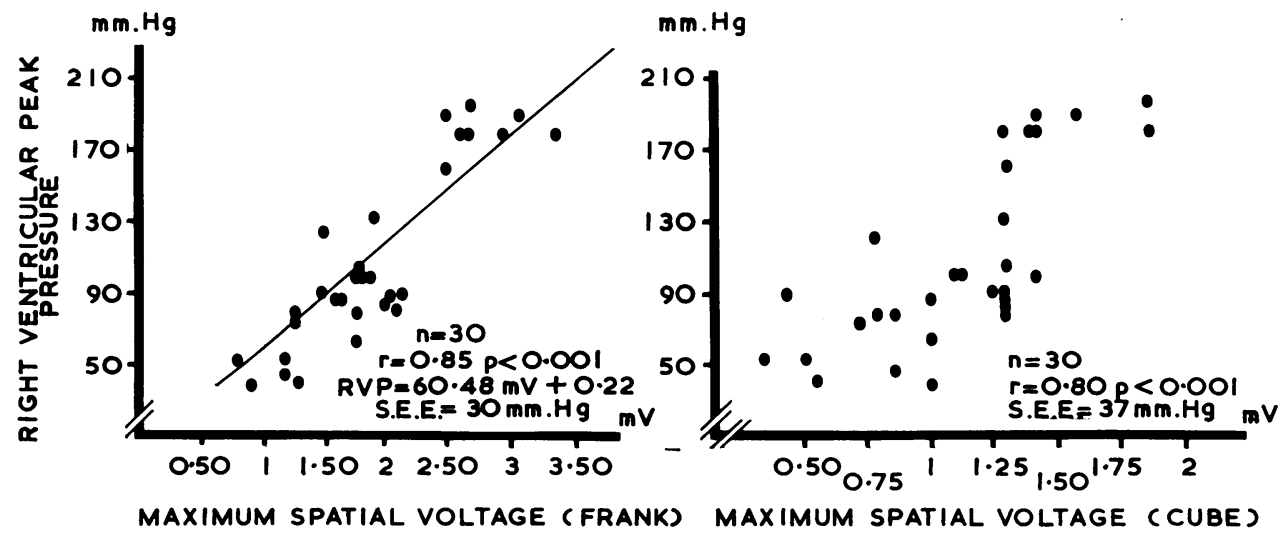

FIG. 1.-Comparison of the correlation between right ventricular peak systolic pressure and the maximum spatial voltage derived by the Frank (left) and cube (right) systems in the same $\mathbf{3 0}$ patients with pulmonary stenosis. 
AND ELECTROCARDIOGRAPHIC DATA

\begin{tabular}{|c|c|c|c|c|c|c|c|c|}
\hline \multicolumn{9}{|c|}{ electrocardiographic data } \\
\hline \multicolumn{4}{|c|}{ Cube } & \multicolumn{5}{|c|}{ Electrocardiogram } \\
\hline \multicolumn{3}{|c|}{ Magnitude of spatial vectors } & \multirow{2}{*}{$\begin{array}{c}\text { Direction } \\
\text { of } \\
\text { rotation }\end{array}$} & \multirow[t]{2}{*}{ RV1 } & \multirow[t]{2}{*}{$\begin{array}{c}\text { Sum of } \\
\text { SI + RV1 + SV6 }\end{array}$} & \multirow[t]{2}{*}{$\mathrm{R} / \mathrm{S}$ ratio $\mathrm{V} 1$} & \multicolumn{2}{|c|}{$\begin{array}{c}\text { Time of occurrence } \\
\text { (Frank) }\end{array}$} \\
\hline Max. & $\underset{\text { ratio }}{\mathbf{L} / \mathbf{R} \mathbf{~ m V}}$ & $\begin{array}{l}\text { Sum of } \\
\text { vectors }\end{array}$ & & & & & MRSV & MSV \\
\hline $\begin{array}{l}1.00 \\
0.54 \\
0.86 \\
0.50 \\
0.34 \\
1.00 \\
0.72 \\
0.79 \\
0.86 \\
1.29 \\
1.29 \\
1.29 \\
1.00 \\
1.29 \\
1.25 \\
0.43 \\
1.41 \\
1.12 \\
1.12 \\
1.31 \\
0.77 \\
1.29 \\
1.31 \\
1.87 \\
1.41 \\
1.39 \\
1.29 \\
1.58 \\
1.42 \\
1.87\end{array}$ & $\begin{array}{l}3.00 \\
1.00 \\
2.00 \\
3.00 \\
1.00 \\
1.50 \\
1.50 \\
1.60 \\
1.10 \\
1.10 \\
1.15 \\
1.00 \\
0.60 \\
0.80 \\
0.90 \\
0.60 \\
1.00 \\
1.10 \\
0.08 \\
0.40 \\
0.80 \\
1.20 \\
0.30 \\
0.70 \\
1.00 \\
0.12 \\
0.17 \\
0.20 \\
0.05 \\
0.90\end{array}$ & 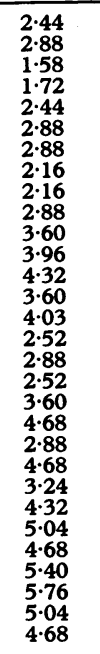 & 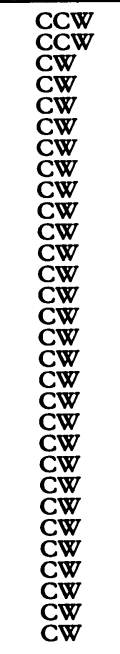 & $\begin{array}{r}1 \\
12 \\
4 \\
4 \\
2 \\
10 \\
11 \\
6 \\
15 \\
4 \\
7 \\
10 \\
10 \\
25 \\
24 \\
18 \\
24 \\
12 \\
12 \\
27 \\
14 \\
10 \\
21 \\
6 \\
10 \\
23 \\
12 \\
30 \\
17 \\
35\end{array}$ & $\begin{array}{r}6 \\
32 \\
35 \\
10 \\
2 \\
35 \\
26 \\
9 \\
20 \\
8 \\
20 \\
30 \\
17 \\
47 \\
21 \\
34 \\
51 \\
25 \\
30 \\
45 \\
27 \\
30 \\
38 \\
18 \\
31 \\
43 \\
30 \\
50 \\
41 \\
59\end{array}$ & $\begin{array}{l}0 \cdot 7 \\
0 \cdot 5 \\
1 \\
4 \\
3 \\
3 \cdot 1 \\
2 \cdot 1 \\
1 \cdot 5 \\
4 \cdot 2 \\
1 \\
7 \\
3 \cdot 2 \\
2 \\
7 \cdot 2 \\
4 \\
2 \\
7 \cdot 2 \\
3 \cdot 6 \\
4 \\
4 \cdot 5 \\
2 \\
4 \\
7 \\
6 \\
5 \\
10 \cdot 1 \\
4 \\
6 \\
17 \\
11 \cdot 1\end{array}$ & $\begin{array}{l}0.07 \\
0.07 \\
0.06 \\
0.06 \\
0.07 \\
0.07 \\
0.06 \\
0.07 \\
0.06 \\
0.06 \\
0.06 \\
0.07 \\
0.07 \\
0.07 \\
0.07 \\
0.06 \\
0.05 \\
0.06 \\
0.08 \\
0.06 \\
0.06 \\
0.07 \\
0.06 \\
0.07 \\
0.07 \\
0.05 \\
0.05 \\
0.04 \\
0.05 \\
0.05\end{array}$ & $\begin{array}{l}0.04 \\
0.04 \\
0.035 \\
0.035 \\
0.04 \\
0.035 \\
0.035 \\
0.035 \\
0.035 \\
0.04 \\
0.04 \\
0.04 \\
0.035 \\
0.04 \\
0.04 \\
0.04 \\
0.035 \\
0.06 \\
0.08 \\
0.06 \\
0.06 \\
0.07 \\
0.06 \\
0.07 \\
0.07 \\
0.05 \\
0.05 \\
0.04 \\
0.05 \\
0.05\end{array}$ \\
\hline
\end{tabular}

pressure; BSA, body surface area.

(Fig. 6). For the Frank data the $r$ value was -0.70 and -0.67 , respectively ( $<<0.001$ for both). The S.E.E. was $0.40 \mathrm{~cm} .^{2}$ and $0.41 \mathrm{~cm}^{2}$ The regression equation for the former was log valve area $=-0.87 \mathrm{mV}+0.46$. For the cube data, $\mathrm{r}$ was -0.62 and $-0.60, p<0.001$, S.E.E. 0.45 and 0.47 $\mathrm{cm} .{ }^{2}$, respectively. These parameters changed little when calculated for the larger group. Using the $S 1+\mathrm{RV} 1+\mathrm{SV6}, \mathrm{r}$ was $-0.48, \mathrm{p}<0.01$, while S.E.E. increased to $0.61 \mathrm{~cm}^{2}$

Correlation with stroke work was poor, $r=0.27$ and $\mathbf{0 . 2 5}$, for Frank and cube data.

The morphology of the horizontal plane projection obtained by the cube method was quite
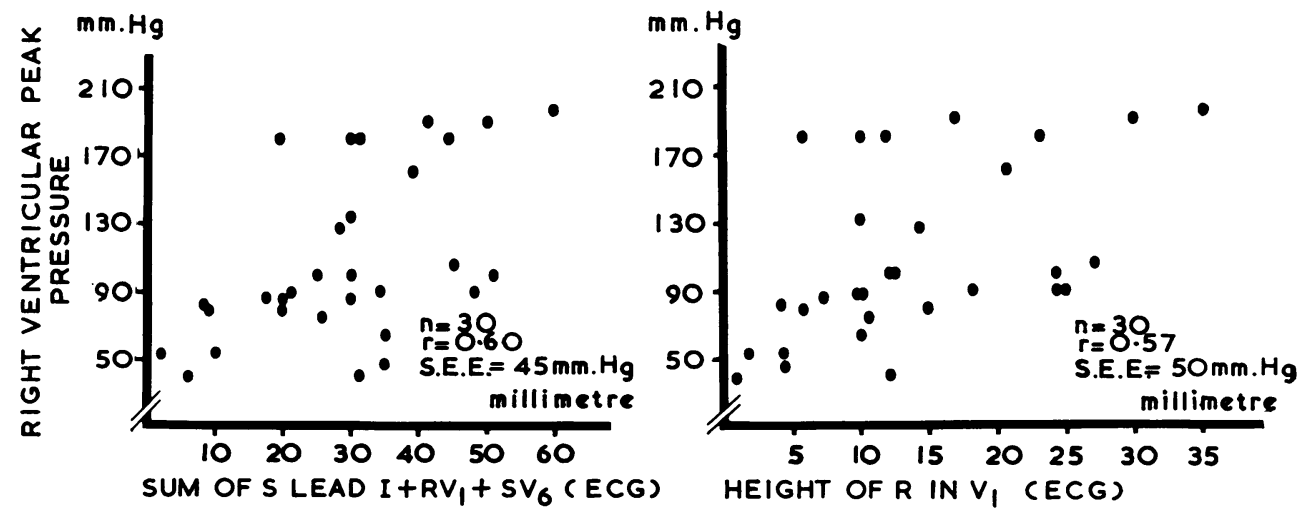

FIG. 2.-Comparison of the correlation between right ventricular peak systolic pressure and two electrocardiographic criteria, $R$ in V1 (commonly used) and the sum of $S$ in lead 1, R in V1, and S in V6 (newly proposed). The improvement with the latter is but slight. 

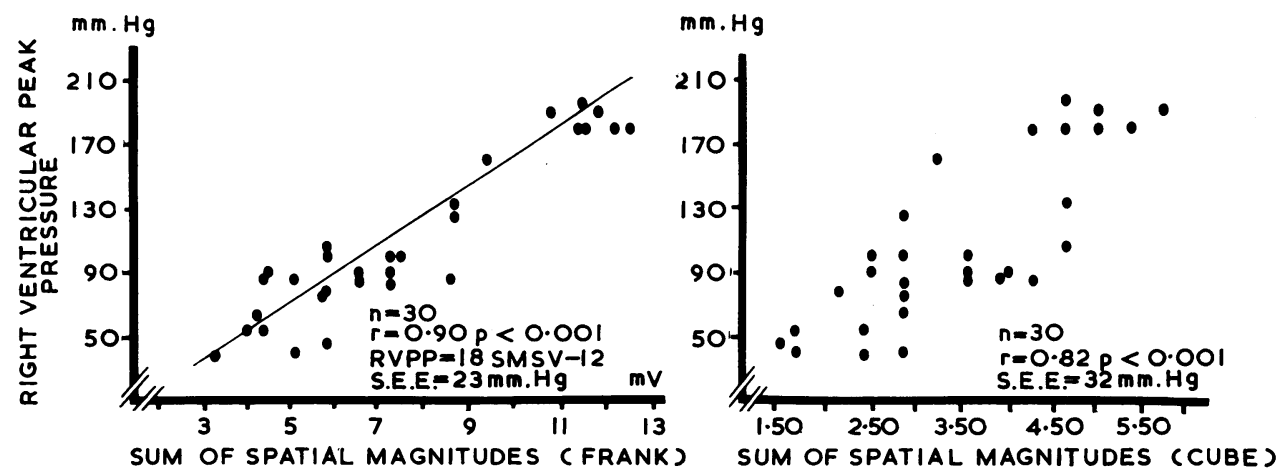

FIG. 3.-The relation of a sum of spatial magnitudes (see text) to the right ventricular peak systolic pressure in the same 30 patients with pulmonary stenosis. Frank system data on left, cube system data on right. The correlation coefficient and the standard error of the mean are the best of any parameters analysed in this study.

stereotyped, all but two showing clockwise rotation in their sequence of depolarization. This generally occurred with a progressively smaller leftward efferent limb as the right ventricular hypertension became more severe. In contrast, 11 of the 30 Frank vectorcardiograms showed counterclockwise progression of the entire horizontal plane loop. In 10 of these, the RVPP was below 80 $\mathrm{mm}$. $\mathrm{Hg}$, while in the eleventh (No. 12) it was $87 \mathrm{~mm}$. Hg (Table I). Clockwise progression occurred in 16 patients, and a narrow loop showing a figure of eight, with posterior orientation, was seen in three. Clockwise inscription was observed only with RVPP above $80 \mathrm{~mm}$. Hg. Illustrations of these discrepancies are given in Fig. 7, 8, 9, and 10.

These ouservations are reflected in the measurements given in the Table and show that right ventricular hypertrophy could be recognized by the standard electrocardiogram by means of the height of the $R$ wave in V1 in all but Cases 1, 3, 4, and 5, by the $R / S$ ratio in all but Cases 1 and 2 and in
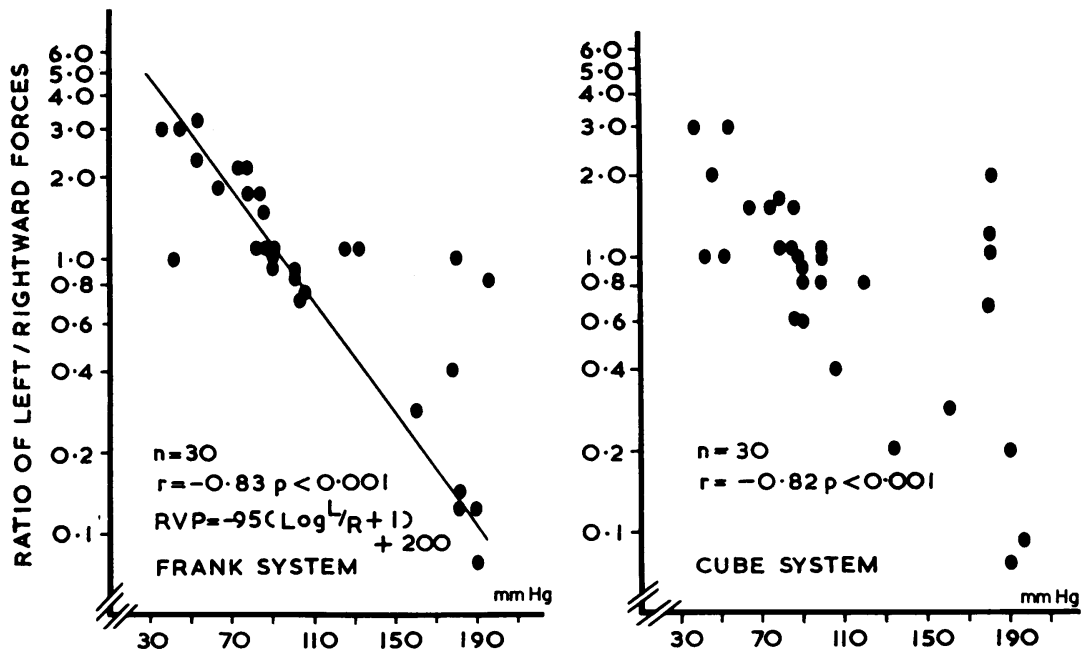

RIGHT VENTRICULAR PEAK SYSTOLIC PRESSURE

FIG. 4.-Relation between the ratio of left/rightward forces as projected on the frontal plane ( $x$ axis) and the right ventricular peak systolic pressure. The results are virtually identical reflecting the fact that the differences in design between the Frank and cube systems influence least the frontal plane display of forces. The findings in the previous figure indicate they mainly affect the horizontal plane display ( $z$ axis). 


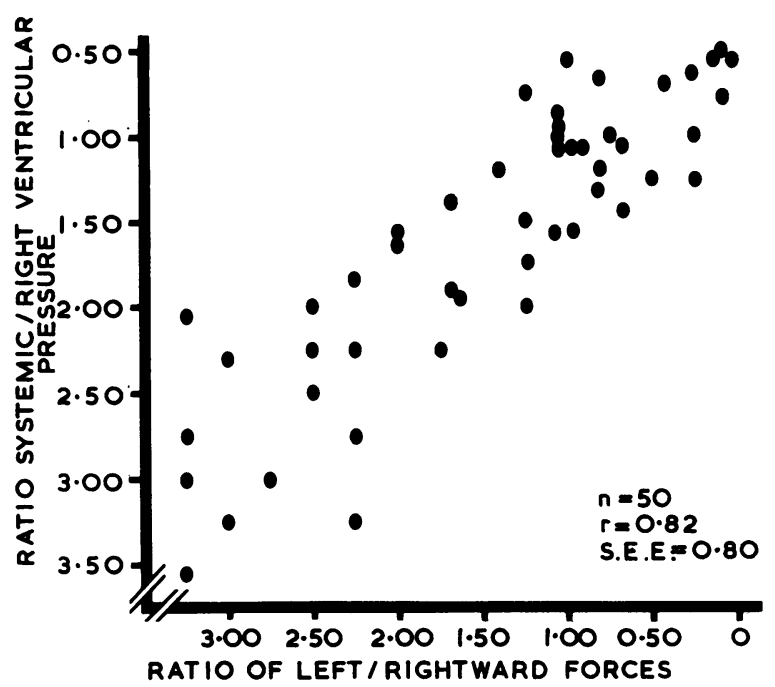

FIG. 5.-Relation of the ratio of systemic to right ventricular pressure to the ratio of electromotive left/rightward forces. This diagram indicates a progressively stronger, rightward deflection of electrical forces with higher right ventricular pressures.

every case by the criterion consisting of the sum of $S$ in $1, R$ in V1, and $S$ in V6. In no instance did the vectorcardiogram, cube or Frank, give qualitative indication of the presence of right ventricular hypertrophy where the electrocardiogram did not, though its presence could be recognized with greater facility by use of the vectorcardiographic criteria.

The time of occurrence of the MRSV obtained by the Frank system varied between 0.038 and 0.080 sec. and was plotted against right ventricular peak systolic pressure (Fig. 11). A distinct trend was seen, patients with mild right ventricular hypertension ( $90 \mathrm{~mm}$. $\mathrm{Hg}$ or less) showing a clear separation between the time of inscription of the MSV (mean at $0.039 \mathrm{sec}$.) and the MRSV (0.069 sec.), while in severer degrees of hypertension, the MSV and MRSV coincided. The majority of these observations fell around $0.07 \mathrm{sec}$., though with

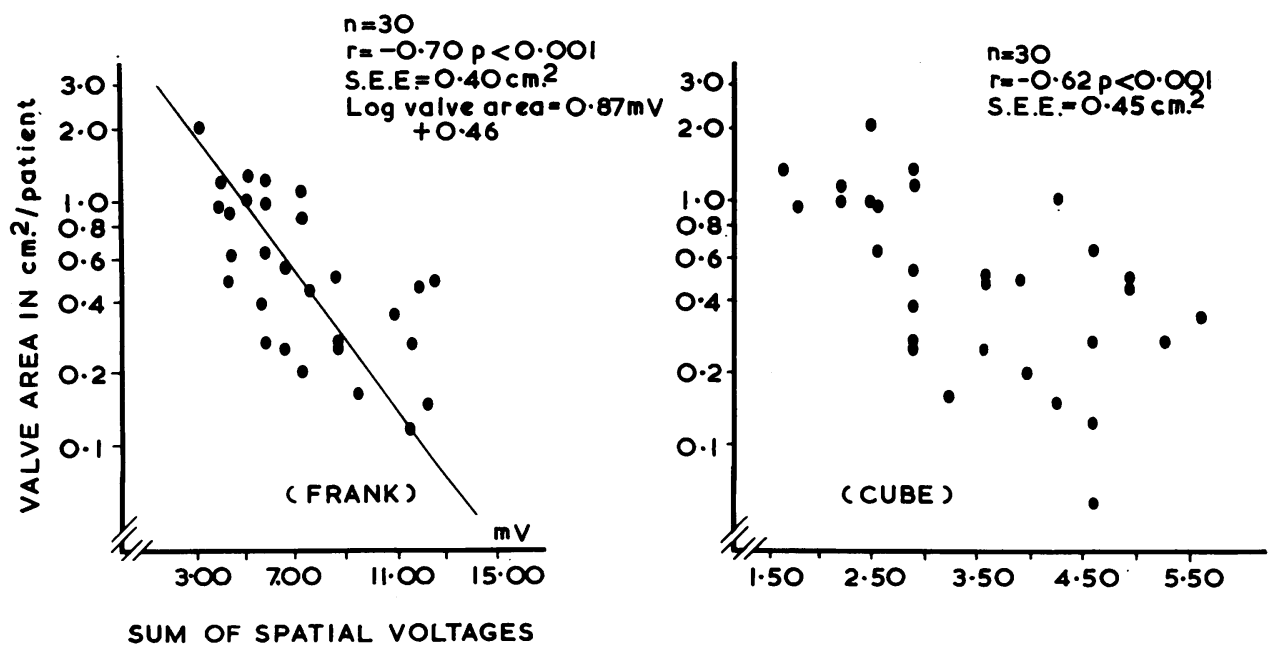

FIG. 6.-The correlation between the "best" vectorcardiographic criterion, a sum of spatial voltages, and the pulmonary valve area in the same 30 patients with pulmonary stenosis. The best coefficient was derived from Frank data, but fell far below those obtained with peak systolic pressure. (Compare Fig. 3.) 


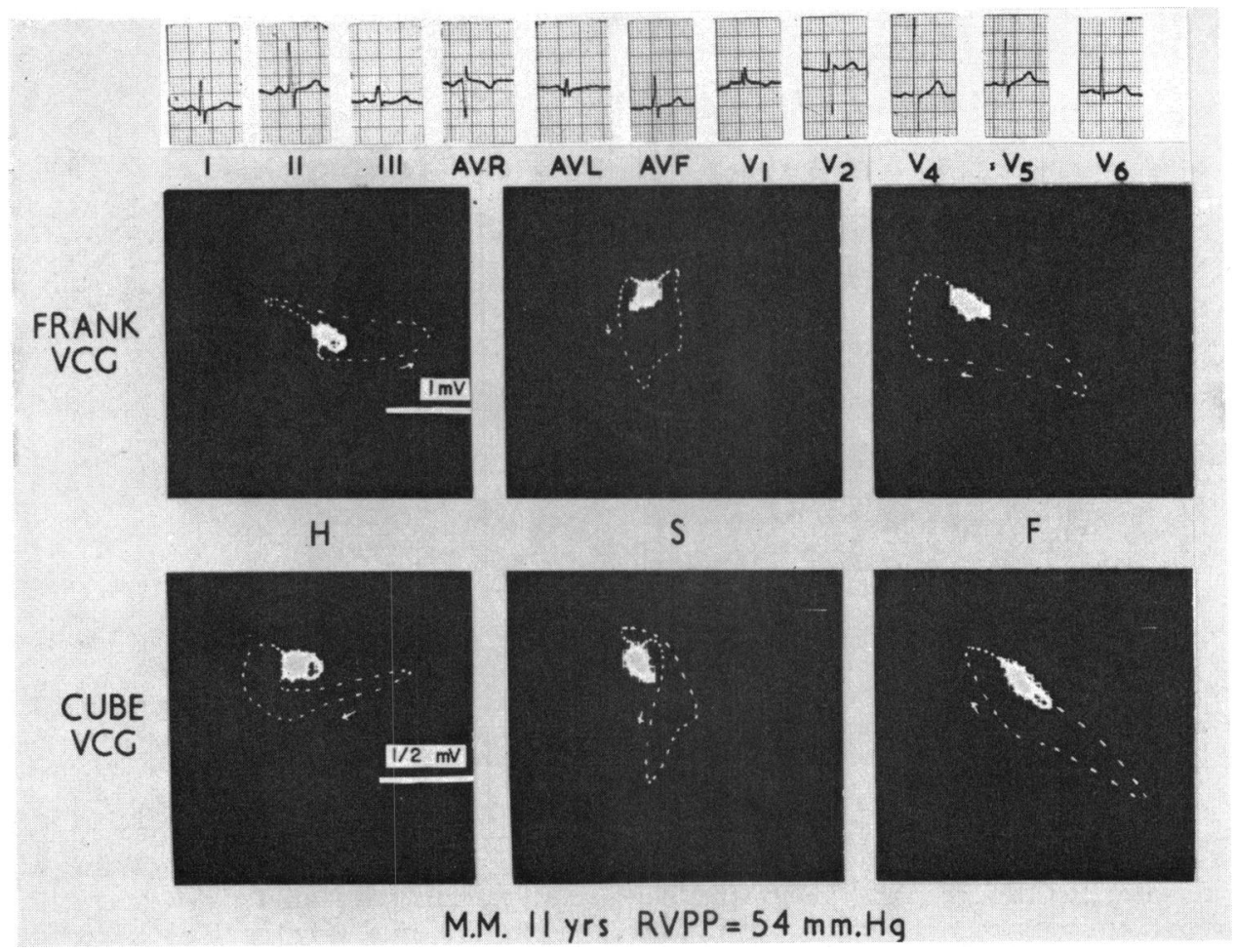

FIG. 7.-Electrocardiogram (upper panel) and vectorcardiograms (lower panels) in a patient with mild, valvular pulmonary stenosis. The Frank system shows augmented spatial voltage in the late QRS forces, whereas the cube system shows rightward deflection of early QRS forces and consequent reversal of rotation to counterclockwise after the 0.03 second vector. The voltage readings of the Frank tracing exceed those of the cube system by a factor or two. In this and the following three illustrations, the Frank sagittal display is from the left, the cube from the right.

very high right ventricular peak pressures the time of the MRSV became again earlier (mean 0.045 sec.).

\section{Discussion}

Recently, the relation between chronically increased pressure as a physiological parameter and the electromotive forces of the myocardium has been explored (Hugenholtz and Gamboa, 1964). It was concluded that the augmented electromotive forces in patients . with hypertrophy were indeed linearly related to a continually increased intraventricular pressure through changes in the histology of the myocardium. In addition it was shown that such electrical forces were not subject to acute changes in myocardial performance and should be considered to reflect the prevalent peak pressure in the ventricle. Since, clinically, the assessment of the physiological state is of more importance than the exact knowledge of heart weight or wall thickness, the application of resting right ventricular peak pressure as the basic parameter, against which the performance of three commonly used lead systems was measured, appears justified.

The data clearly show the superiority of the orthogonal lead system of Frank $(r=0.90)$ over the cube system $(r=0.83)$ and the standard electrocardiogram $(r=0.60)$, even when the "best" criteria [SMRSV, and S1 + RV1 + SV6] are applied (Fig. 1, 2, and 3). The difference between lead systems, in their assessment of intraventricular pressure, is even more obvious in the degree of scatter of individual measurements as expressed by the standard error of estimate. Even for the larger group of patients, the S.E.E. obtained by the Frank system was less than half that obtained by the electrocardiogram (20 v. $50 \mathrm{~mm}$. $\mathrm{Hg}$, while in the cube system S.E.E. was $30 \mathrm{~mm}$. Hg). Thus, the maximal spatial magnitude, or the sum of spatial magnitudes at the time the right ventricle exerts its dominant influence, may be introduced as a new criterion for the vectorcardiographic estimation of hypertrophy. Provided it is obtained with an 


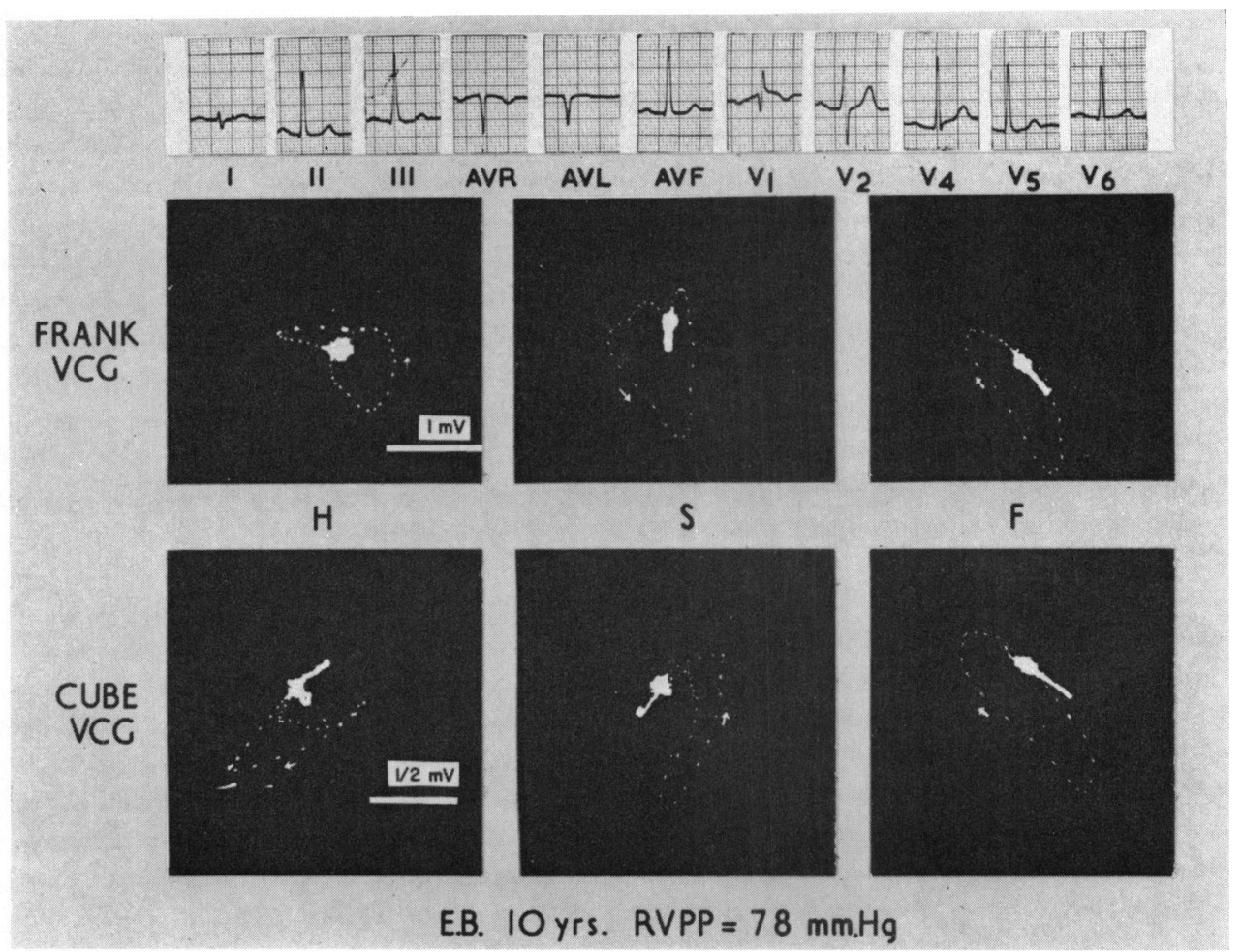

FIG. 8.-Tracings in a patient with moderate right ventricular hypertension. The same difference in volt ages and direction of rotation exists as in the previous tracing. In addition, the amount of leftward directed forces is decreased in the Frank tracing and that of anteriorly directed forces increased in the cube tracing.

orthogonal lead system, it permits a degree of accuracy hitherto unknown in the estimation of right ventricular pressure in pulmonary stenosis. An even greater degree of accuracy, particularly in patients whose right ventricular pressure is below the systemic pressure, can be obtained by combining the SMRSV information with that provided by the $L / R$ ratio of forces projected on the axis (compare Fig. 3 and 4).

Correlations with pulmonary valve area were less significant (Fig. 6) and those with stroke work were insignificant. These findings are in keeping with the concept (Monroe, 1964) that the production of peak pressure is, metabolically speaking, the most "costly" performance of the heart and is the dominant factor leading to anatomical changes. Since calculation of pulmonary valve area and stroke work reflects displacement of stroke volume, myocardial function may vary widely without influencing myocardial oxygen consumption or myocardial histology. Thus, it is not surprising to find a less satisfactory correlation with these parameters (Haywood, Selvester, and Griggs, 1961). A satisfactory, though non-quantitative, correlation of stroke work with the cube vectorcardiogram has been found, however, in atrial septal defects (Liebman and Nadas, 1960), a lesion where augmentation of stroke volume may become so large that it exceeds the influence of pressure in the calculation of stroke work. It should be stressed, therefore, that the correlations found in the present study apply only to the simplified "model" of pure pulmonary stenosis (valvar) with concentric hypertrophy, where factors such as dilatation of the right ventricle are not present and stroke volume and stroke work vary but little.

The discrepancies in the performance between both vectorcardiographic systems, already predicted on the basis of theoretical considerations (Pipberger and Lilienfield, 1958; Brody and Arzbaecher, 1964), are even more striking in view of the continuing popularity of the cube system. In addition, as the present study demonstrates, wide variations in the morphology of the spatial vectorcardiogram derived by these systems have been observed. The reasons for these differences in correlations with hæmodynamic data and in their 


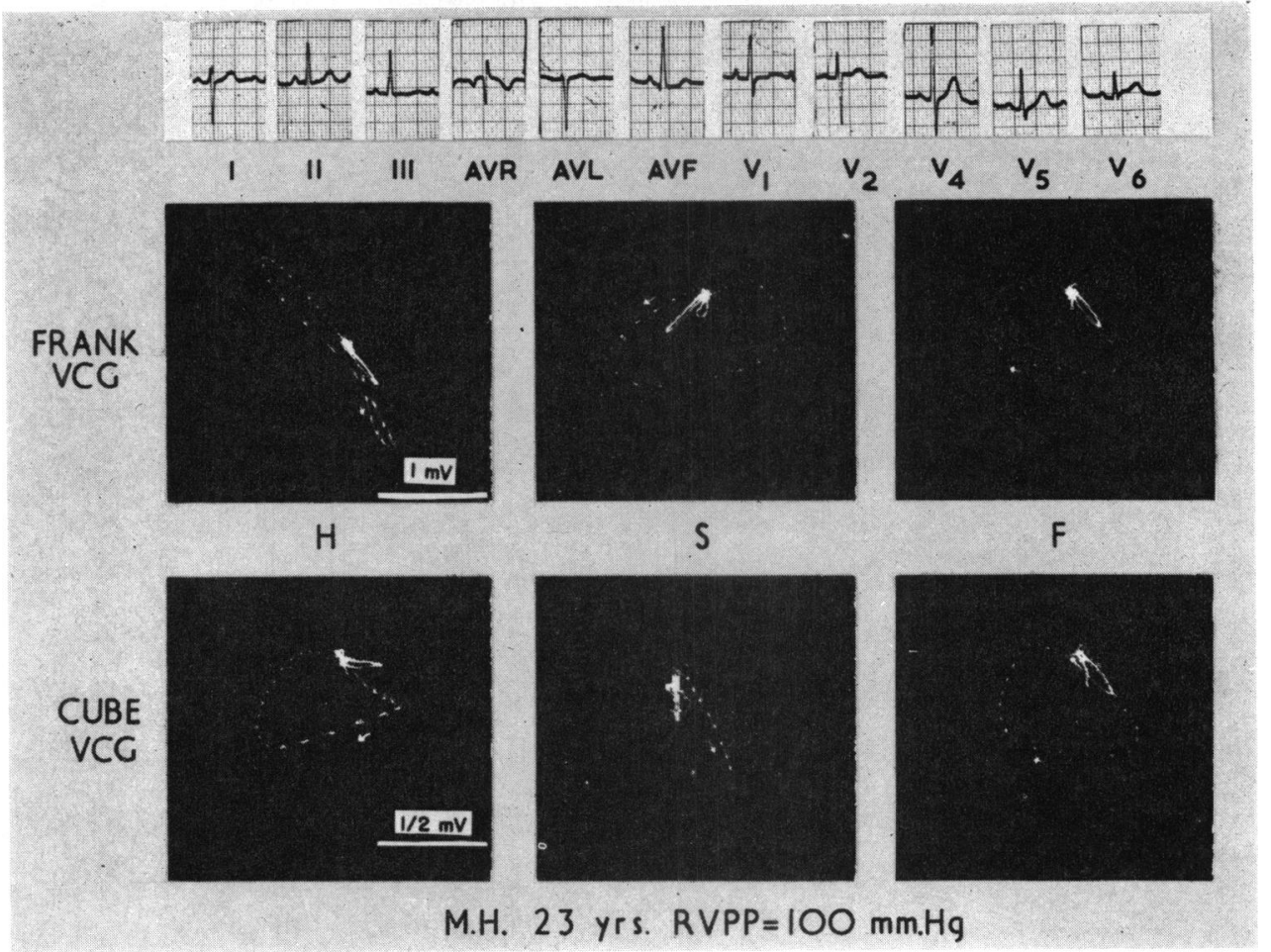

FIG. 9.-Tracings of a patient with moderately severe right ventricular hypertension. The horizontal plane display of the cube tracings shows little change from the previous or the next illustration, whereas that derived by the Frank system shows marked differences both in the magnitude and direction. All patients with pressures in excess of $80 \mathrm{~mm}$. $\mathrm{Hg}$ showed counterclockwise rotation in the horizontal plane in the Frank system recordings.

morphological appearance seem to be also related to the manner in which both systems record the changing dipoles occurring in right ventricular hypertrophy.

In the absence of right ventricular conduction defects (Milnor, 1957), the development of right ventricular hypertrophy may be expected to influence the balance with left ventricular forces in a linear fashion. Early in this process more initial QRS forces will escape the left ventricular cancellation effect (Cohen, Abildskov, and Jacobson, 1961) and become oriented anteriorly. Simultaneously with this, late QRS forces may increase in magnitude without much change in their orientation. With further increases in right ventricular mass, more QRS forces may deviate to the right and the resultant QRS forces in the mild portion of the QRS complex may deflect either anteriorly or posteriorly (Strang et al., 1963). Eventually, when right ventricular mass significantly exceeds that of the left ventricle, all QRS forces, early, mid, and late will deviate anteriorly and to the right.
This process was observed unusually early in the records obtained by the cube system. In fact, mild degrees of right ventricular hypertension (associated with prefectly normal heart size by radiograph, Cases 3-9, Table) have led in each instance to a complete reversal of the inscription in the horizontal plane cube vectorcardiogram (Fig. 7 and 8). This occurred at a time when systemic, and thus left ventricular pressure, was in excess of 105 $\mathrm{mm}$. $\mathrm{Hg}$, while right ventricular pressures were less than $80 \mathrm{~mm}$. Hg. Although such reversal of inscription is obviously an easily recognizable feature in the tracing, the actual hæmodynamic balance of right and left-sided forces was still strongly in favour of the latter. Since a direct and close relation between hæmodynamic factors and electromotive forces has been proven (Hugenholtz and Gamboa, 1964), it could be expected that in these instances a leftward and counterclockwise progression of depolarization forces would prevail as long as left ventricular pressures exceeded right ventricular pressures. Such counterclock- 


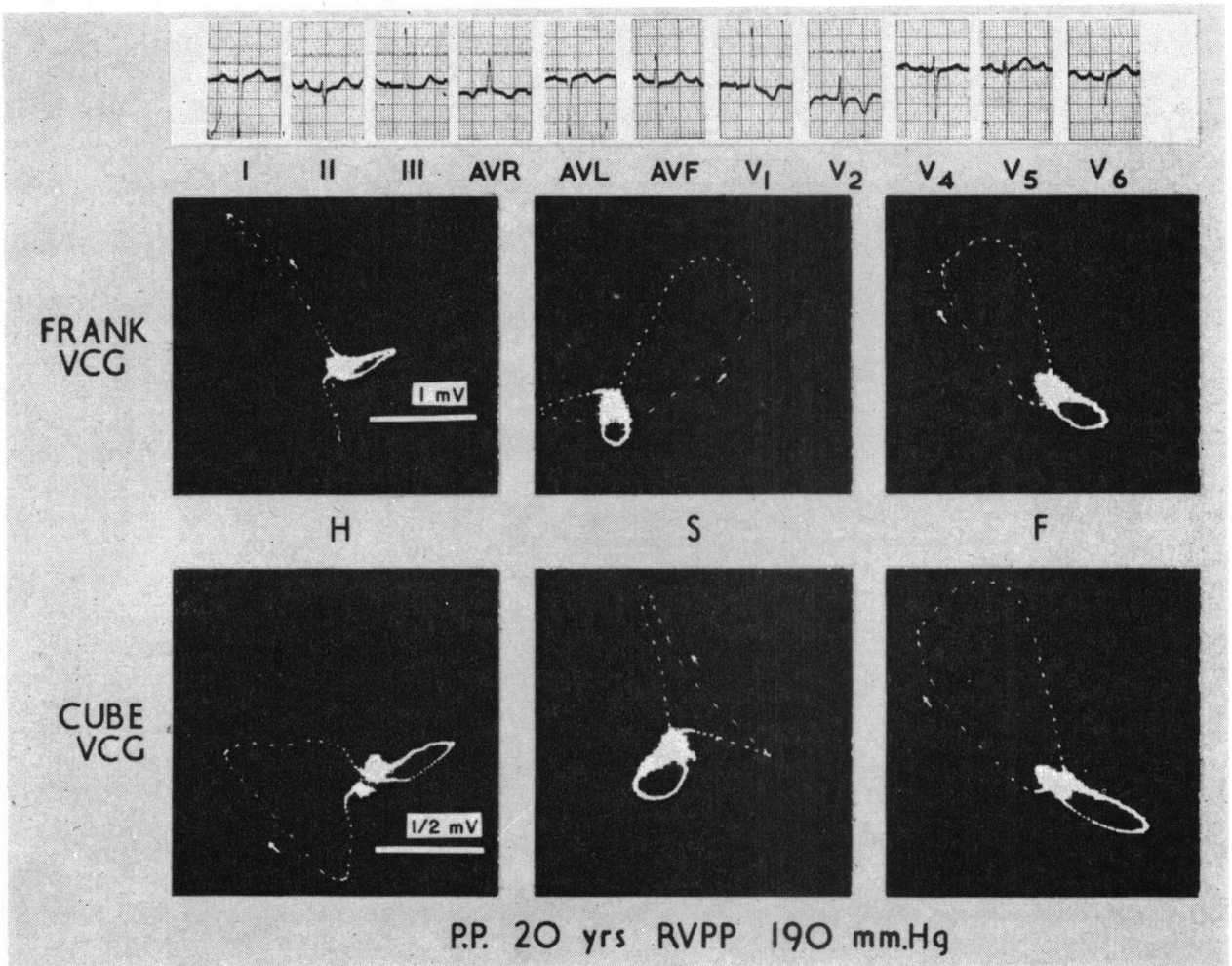

FIG. 10.-Except for a further increase in the spatial magnitude of the maximum rightward vector, the tracings of this patient with severe right ventricular hypertension show little change from the recording in the previous illustration. In this and all previous recordings, the greatest discrepancy between the systems was found in the horizontal plane display and the least in the frontal plane display.

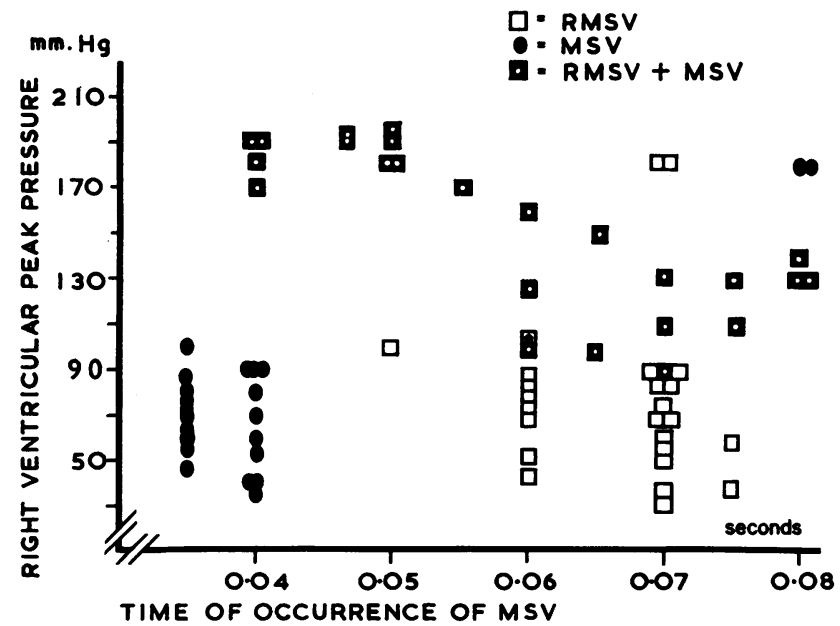

Fig. 11.-Plot of the time of occurrence of the maximum spatial vector (right and left) against peak ventricular pressure. When the pressure was in excess of the systemic pressure range, the maximum spatial vector was invariably to the right and with higher pressure occurred progressively earlier, indicating greater capability of cancellation and domination over left ventricular forces. In patients with RVPP below this range, the RMSV was late and occurred at a different time from the leftward directed MSV. 


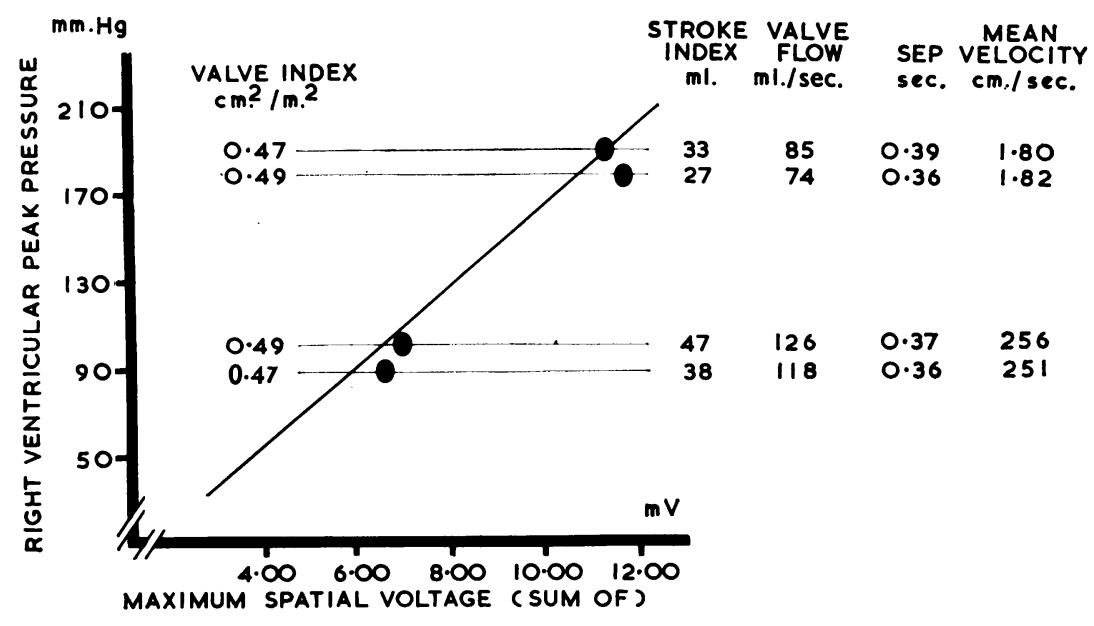

FIG. 12.-The clinical significance of the SMRSV in the assessment of right ventricular hypertension is given by 4 patients with similarly restricted valve areas. While the different levels of right ventricular pressures are explained in part by varying stroke index and valve flow, the greatest significance was attached to differences in mean velocity of blood flow across the pulmonary valve under resting circumstances. Since the velocity was calculated to be much faster in the two lower examples, it required lower peak pressures and resulted in lower spatial voltages.

wise progression in the face of mild-to-moderate right ventricular hypertension in fact was found in the Frank vectorcardiogram of these same patients (Fig. 7 and 8 ).

Recent studies of Taccardi (1963) and Horan, Flowers, and Brody (1963) have shown that uncorrected præcordial leads, such as are used in the cube system and in the standard cardiogram, are not dipolar throughout the entirety of ventricular depolarization. Such non-dipolar behaviour of the præcordial leads in the cardiogram appears to reflect the proximity of the right ventricle and its multipolar effect, particularly when they are increased, rather than the proportional contribution of these to the average heart dipole. Since recording systems that purport to record spatial vectorcardiograms are based on the principle that the heart can be represented by a single dipole (Frank, 1956), this kind of discrepancy between the configuration of the horizontal loop derived from standard præcordial leads from the cube system, when compared with that obtained by the Frank system, clearly demonstrates that the "uncorrected" lead systems do not adhere to the dipole concept (Sodi-Pallares et al., 1961). The result of this fact may indeed be a maximal sensitivity in the detection of increased right ventricular forces, but at the same time it leads to a lack of specificity in the estimation of the degree of right ventricular hypertension.

With the Frank lead system, augmentation of the maximal spatial forces occurring to the right appears to be the simplest, though not the only, criterion for quantitation of right ventricular hypertrophy. In mild right ventricular hypertrophy, these vectors are late, at $0.07 \mathrm{sec}$., occurring at a time when the left ventricle has virtually terminated its depolarization, and are relatively small, as the right ventricle is not much hypertrophied and may have thickened in its outflow portion only (Fig. 7). In keeping with this, the occurrence of the maximum spatial vector (MSV), which in this group is still directed leftward (Fig. 7 and 8), is much earlier, at $0.04 \mathrm{sec}$., while its magnitude is also much larger (Fig. 11). When the degree of right ventricular hypertension increases, this difference in timing of the MRSV and MSV disappears. In the patients in whom right ventricular peak pressure was equal to or exceeded systemic pressure, the magnitude of the MSV increased further, its direction becoming either more anterior or posterior, but rightward (Fig. 9 and 10). Eventually, right ventricular forces entirely dominate the depolarization process, presumably because all areas of the right ventricle have hypertrophied, and all QRS forces are oriented in a rightward direction. This sequence in the increase of the magnitude of the MRSV is much more in keeping with the anatomical changes in progressively more severe degrees of right ventricular hypertension than the one found in cube recordings. 
From a clinical point of view these findings suggest that the presence of right ventricular hypertrophy could be detected in each case by the standard electrocardiogram or the cube system through the criteria applied in this study. On the other hand the Frank vectorcardiogram proved its superiority in indicating quantitatively the degree of right ventricular hypertension. Thus for the clinician, a combination of the standard cardiogram for recognition and the corrected vectorcardiogram for measurement appears advisable in the assessment of right ventricular hypertension.

Although no comparable quantitative studies relating orthogonally-derived electrocardiographic data to hæmodynamic events have been published so far, it is of some interest to compare our findings with those published from standard electrocardiographic data. The most comprehensive study in this regard was published by Bassingthwaighte et al. (1963). These workers found a so far unequalled correlation between the log of peak voltage in lead $\mathrm{V} 1$ and the $\log$ of pulmonary valve area $(\mathrm{r}-0.79)$, while a less satisfactory correlation was demonstrated $(r=0.63)$ with peak gradient. In a larger number of patients, Cayler, Ongley, and Nadas (1958) established a coefficient of 0.78 between peak pressure and R in V1. While these correlations, in general, are not as good as those obtained by the Frank system, they point to the fundamental question whether pressure requirement alone or total outflow resistance is the determining factor for the development of compensatory histological changes. This may be illustrated by Fig. 12 where, in 4 patients with comparable valve areas, sharply differing peak pressures were required under the same resting circumstances to establish an effective pulmonary flow. These differences may be explained by the individual adjustments of resting stroke volume and the velocity of valve flow. The latter in turn may depend on the "efficiency" of the ejection, on the internal architecture of the right ventricular outflow tract, and on the presence of muscular obstruction in this area, settings that may vastly differ from individual to individual. Since the peak pressure predicted from the spatial voltages in each of these 4 patients correlated very well with the observed measurements under resting circumstances, the requirements for and the "setting" of peak pressure appear to be the main factors, overriding valve size (per se) in significance. Furthermore, this conclusion is in keeping with previous work, showing that peak systolic pressure remains the most important determinant of myocardial oxygen consumption (Monroe, 1964) as well as the main cause of changes in myocardial structure, if requirements are chronically augmented (Linzbach, 1960).

\section{SUMMARY}

The results of the standard electrocardiogram, cube vectorcardiogram, and the corrected vectorcardiogram derived by the Frank system, in 50 patients with valvar pulmonary stenosis were compared with hæmodynamic data obtained at cardiac catheterization.

Right ventricular hypertension at rest was found to range from 40 to $196 \mathrm{~mm}$. Hg. Right ventricular peak pressure, right ventricular work, and pulmonary valve area were used as objective criteria for comparison. Two new vectorcardiographic parameters (MRSV and SMRSV) were derived, based on the measurement of the maximum rightward directed spatial force. Standard electrocardiographic criteria were employed.

A significant correlation between right ventricular peak pressure and the maximum rightward spatial vector $(r=0.87)$ and a sum of maximum rightward vectors $(r=0.90)$ was found, using data derived from the Frank system. Similar correlations for the cube system were less significant $(r=0.82$ and 0.83 , while all correlations with ventricular work were insignificant.

These results indicate the superiority of the orthogonal corrected lead system of Frank in these electrocardiographic-hæmodynamic correlations. The progressively changing configuration of spatial forces with increasing severity of hypertension gives further insight into the alterations in the depolarization process in right ventricular hypertrophy. These findings also prove that, with new criteria employing the measurement of truly spatial magnitudes, reliable estimation of resting right ventricular pressure may be obtained.

\section{REFERENCES}

Bassingthwaighte, J. B., Parkin, T. W., DuShane, J. W., Wood, E. H., and Burchell, H. B. (1963). The electrocardiographic and hemodynamic findings in pulmonary stenosis with intact ventricular septum. Circulation, 28, 893.

Brody, D. A., and Arzbaecher, R. C. (1964). A comparative analysis of several corrected vectorcardiographic leads. Circulation, 29, 533.

Cayler, G. G., Ongley, P., and Nadas, A. S. (1958). Relation of systolic pressure in the right ventricle to the electrocardiogram: A study of patients with pulmonary stenosis and intact ventricular septum. New Engl. F. Med., 258, 979.

Cohen, W., Abildskov, J. A., and Jacobson, E. D. (1961). Theoretical and clinical studies of the electrocardiogram and vectorcardiogram in right ventricular enlargement. Amer. Heart F., 61, 656.

Donoso, E., Sapin, S. O., Braunwald, E., and Grishman, A. (1955). A study of the electrocardiogram and vectorcardiogram in congenital heart disease. II. Vectorcardiographic criteria for ventricular hypertrophy. Amer. Heart F., 50, 674. 
Duchosal, P. W., and Sulzer, R. (1949). La Vectocardiographie. Karger, Bâle.

Frank, E. (1956). An accurate, clinically practical system for spatial vectorcardiography. Circulation, 13, 737.

Grishman, A., and Scherlis, L. (1952). Spatial Vectorcardiography. W. B. Saunders, Philadelphia.

Haywood, L. J., Selvester, R. H., and Griggs, D. E. (1961). Correlations of right ventricular work with the vectorcardiogram in pulmonary stenosis (Abstract). Clin. Res., 9, 62.

Horan, L. G., Flowers, N. C., and Brody, D. A. (1963). Body surface potential distribution. Circulat. Res., 13, 373.

Hugenholtz, P. G., and Gamboa, R. (1964). Effect of chronically increased ventricular pressure on electrical forces of the heart. A correlation between hemodynamic and vectorcardiographic data (Frank system) in 90 patients with aortic or pulmonic stenosis. Circulation, 30, 511.

Liebman, J., and Nadas, A. S. (1960). The vectorcardiogram in the differential diagnosis of atrial septal defect in children. Circulation, 22, 956.

Linzbach, A. J. (1960). Heart failure from the point of view of quantitative anatomy. Amer. F. Cardiol., 5, 370.

McFee, R., and Parungao, A. (1961). An orthogonal lead system for clinical electrocardiography. Amer. Heart f., 62, 93.

Milnor, W. R. (1957). Electrocardiogram and vectorcardio- gram in right ventricular hypertrophy and right bundlebranch block. Circulation, 16, 348.

Monroe, R. G. (1964). Myocardial oxygen consumption during ventricular contraction and relaxation. Circulat. Res., 14, 294.

Pipberger, H. V., and Lilienfield, L. S. (1958). The application of corrected electrocardiographic lead systems in man. Amer. F. Med., 25, 539.

Schmitt, O. H., and Simonson, E. (1955). The present status of vectorcardiography. Arch. intern. Med., 96, 574.

Scott, R. C. (1960). The correlation between the electrocardiographic patterns of ventricular hypertrophy and the anatomic findings. Circulation, 21, 256.

Sodi-Pallares, D., Bisteni, A., Testelli, M. R., and Medrano, G. A. (1961). Ventricular activation and the vectorcardiogram in bundle-branch blocks. Circulat. Res., 9, 1098.

Strang, R. H., Hugenholtz, P. G., Liebman, J., and Nadas, A. S. (1963). The vectorcardiogram in pulmonary stenosis: Correlation with the hemodynamic state in patients with and without ventricular septal defect. Amer. F. Cardiol., 12, 758.

Taccardi, B. (1963). Distribution of heart potentials on the thoracic surface of normal human subjects. Circulat. Res., $12,341$.

Wilson, F. N., Johnston, F. D., and Kossmann, C. E. (1947). The substitution of a tetrahedron for the Einthoven triangle. Amer. Heart $\mathcal{F} ., 33,594$. 